\title{
Spinal epidural hematoma in hemophilic children: controversies in management
}

\author{
Armando Cama
}

Published online: 10 April 2009

(C) Springer-Verlag 2009

Spinal hemorrhagic complications in hemophilic children are rare but well known. Emergent presentation includes (1) patients with known hemophilia, in whom the diagnostic assessment is easier, and (2) patients without a prior diagnosis, in whom coagulation factor imbalances should always be actively sought. After the presence of an epidural hematoma has been established based on clinical and neuroimaging findings, the most urgent intervention is to normalize the coagulation status. If the hematoma is small or symptoms are mild, clinical, neurophysiological, and magnetic resonance imaging (MRI) monitoring should be performed with the aim to avoid, if possible, surgical procedures. On the contrary, when signs of progressive spinal cord compression are observed, surgery becomes mandatory. A limited laminotomy should be performed whenever possible in order to prevent postsurgical spinal instability. To this end, we suggest to place a cutaneous mark under MRI guidance, thereby allowing for a minimally invasive approach. Factor VIII restoration therapy should be continued until 1 week after surgery. Timely diagnosis and multidisciplinary treatment allow to achieve complete recovery in the vast majority of patients. 